\title{
Fuzziness, Cognition and Cybernetics: a historical perspective
}

\author{
Rudolf Seising ${ }^{1,2}$ Marco Elio Tabacchi ${ }^{3,4}$ Settimo Termini $^{1,3}$ Enric Trillas $^{1}$ \\ ${ }^{1}$ European Center for Soft Computing, Mieres (Asturias), Spain \\ ${ }^{2}$ Friedrich-Schiller-Universität, Jena (Germany) \\ ${ }^{3}$ Dipartimento di Matematica e Informatica, Università di Palermo (Italy) \\ ${ }^{4}$ Istituto Nazionale di Ricerche Demopolis (Italy)
}

\begin{abstract}
In the present paper, we connect some old reflections about the relationships existing between the theory of fuzzy sets and cybernetics with modern, contemporary analyses of the crucial (better: unavoidable) role that fuzziness plays in the attempts at scientifically describing aspects of information sciences. The connection, which has a basic conceptual origin, has been triggered also by the recent 50th anniversary of Norbert Wiener's death which has been instrumental in looking again at some crucial aspects of the birth of information sciences in the midst of the last century. Fuzzy sets are an essential part of this revolution and share all the innovations as well as the difficulties of this towering scientific enterprise which has changed the vision of what a scientific approach must be when dealing with something like information so different from the old matter and energy.t These considerations are helpful in looking in an enlarged way at how to treat and consider the notion of cognition.
\end{abstract}

Keywords: cybernetics, fuzzy set, fuzziness

\section{Introduction and motivations}

In this paper we shall present and discuss some foundational aspects of fuzzy sets [1] by looking at connections between various aspects of the "informational" scientific revolution which took place in the middle of the last century, involving in the first place the birth and the first evolution of Cybernetics. A reflection about the consequences of these connections for the future of the disciplines involved will be made in [2].

\subsection{The rediscovering of old connections}

The fact that last year has been remembered the 50th year of the death of Norbert Wiener is the occasion to look again at some very deep facts that have characterized innovative ways at looking at the scientific enterprise and its impact on society.[3] This connection seems particularly meaningful and interesting to us mainly, of course, for its conceptual import and the possibilities it offers to look at the question posed by the emergence of the notion of fuzziness as a new scientific notion in this 50th anniversary. In some (more or less) recent papers of historical reconstruction of ideas $[4,5]$ it has been underlined the fact that this conceptual milieu has been of crucial importance and interest for the birth of these same ideas.

\subsection{Structure of the paper}

The structure of the paper is as follows: In Section 2 we shall summarize some of the critical analyses specifically done on Cybernetics looking for indications we can have for present days questions and problems. In Section 3 a synthesis of the historical analyses of the foundational connections existing between the "cybernetic thought" and the basic motivations of fuzzy sets will be provided.

A provisional set of conclusions will follow, in order to stimulate the discussion of the future outlook for the disciplines involved.

\section{The cybernetic roots of information sciences (and of fuzzy sets)}

In the present section, the attempt will be done of reading again - in the light of the developments of the past 30 years - what in some old papers has been argued with respect to the status and the problems of Cybernetics and its relations to other related fields and theories, like the, then very young, theory of fuzzy sets. The desire of looking at these old analyses and reflections has not the aim of indulging in the mythologizing the past but, on the contrary, by comparing old attempts with more recent and mature reflections, to try to understand better the dynamics of scientific thought in the new emerged and still emerging fields related to the handling of information. The final and central aim of this enterprise is to search for the better and more promising avenues for present day investigations.

\subsection{An early attempt}

Let us now try to synthetize the first analysis done on these connections. Let us remember that this analysis belongs to a period in which Cybernetics began to disappear and fuzzy sets had just begun to grow ${ }^{1}$. It is not

\footnotetext{
${ }^{1}$ The first reflections on these connections belongs, in fact, to informal exchanges between one of the authors and Joe Goguen at the end of the Seventies of the last century. Their first presentation to a wider audience was in a seminar in Barcelona (Spain), under Enric Trillas's invitation in 1980, as is remembered in [6]. Its first publication al-
} 
a case, then, that this first written paper [7] was entitled "Do Cybernetics, System Science and Fuzzy Sets share some epistemological problems?" 2 The core of the analysis done in it was very bitter and trenchant for Cybernetics. Although very innovative in its content and in its methodology it was stated there that it was very difficult to assess the role that this discipline had played as such (that is: "as a scientific discipline") and from the point of view of specific scientific results: "It seems difficult to give a precise characterization of the role played by $\mathrm{Cy}-$ bernetics. Indeed, one has to consider both its innovative program and the fact that it faded away rather quickly as a specific scientific discipline. Although Cybernetics was quite relevant for the beginning of new disciplines (...) it is hard to point out any specific result which could contemporarily be considered both at its level of generality and strictly pertaining to its intended domain" (page 460).

\subsection{A more mature reflection}

Let us now see how the same question is considered a few years later [8]: "In the middle of the last century a few scientific fields of investigation which dealt (in various ways and in different degrees) with the processing of information raised an enormous number of challenging and innovative problems as far as accepted scientific tradition was concerned. At the same time (and this is the paradox which is worthwhile investigating) all the results that emerged from these investigations were unable to manifest themselves as a consolidated whole, that is, to present themselves in an organized, unitary way which would legitimize the birth of a "single", scientific discipline, with its specific features, methods and results, that would clearly differentiate it from other mainstream disciplines. This situation - common to a set of interestingly different investigations that took place under differing names - is, in our opinion, paradigmatically reflected in the development of Cybernetics“.

By looking for the cybernetics roots of fuzzy sets and information sciences, we are putting into evidence that what is new and important in these fields is that we are constructing an experimental science. A domain, however, in which there is no matter and no energy, but only "information", whatever this name will stand for. Strangely enough, in fact, in this new domain there is nothing that is usually related and associated to experimental sciences: in fact we are dealing with "the immaterial" [9]. We could also say that we are constructing a "physics of the immaterial". A correct definition, if we go to the greek root of the the word Physis, Nature. In nature we have also "immaterial aspects", the information, and we must treat these natural aspects with the same approach and the same methodology of classical physics and biology and all that, but taking into account

\footnotetext{
ready quoted owes its origin to an invitation of Ron Yager to write down these ideas and present them in one meeting he was organizing.

${ }^{2}$ The subtitle ("I. An analysis of Cybernetics") clearly indicates that there was the plan to go on a systematic analysis along the same lines. A program that was not pursued, although many reflections and comments had been done, along the years, but not in a systematic way.
}

the specificity of the new notion in order to treat it in the best possible way.

\subsection{Relevance for present day investigations}

Rethinking the same questions and problems a few decades later, we saw the problem of the rise and decline of Cybernetics as an innovative paradigm and also as a new discipline, in a different way. Let us summarize some of the points. We had to recognize that Cybernetics did also something different and equally if not more important and profound. It indicated that the new scientific remarks and results that were developing around the notion of information and which as told before could also be called, as seen as a sort of a "physics of the immaterial" were - naturally re-aggregating and restructuring around a new paradigm. And this paradigm is now becoming the standard paradigm of this new (macro) disciplinary Sector. It is also becoming an accepted and respectful reference for the development of science in general, and differently from what happened along the years of the decline of Cybernetics as an accepted new discipline, it is considered one of the way according to which science develops.

The novelty, with respect to a few decades ago, is that this kind of development is considered part of the scientific tradition. And that is a crucial point. It is an innovation with respect to the fashion and the mood of a few decades earlier, the ones predominant in the major part of last century, but recovering the very useful interaction between informal notions and their possible formalization in a dynamic way and not "once and for all", as the standard trend of foundational investigations of the last century induced to think. An interesting connection between these attitude and the subsequent structuralist turn in mathematics, of which Bourbaki's attempt is the typical example, should, perhaps be examined for what our questions are concerned. Every foundational analysis should be seen as an interesting way of understanding very subtle questions, but not as the "definitive" and final solution of the same questions. So, the history of Cybernetics must be seen in a different way, as the slowly emerging paradigm of a more complete and faceted way of doing research in a informational age.

Also the warnings towards unproductive developments of fuzzy sets have changed and must change. While in the ' 80 - by correctly doing the comparison with Cybernetics's fate - we were warning against an uncritical use of the new interesting notion, we now consider that the crucial point is to look for new domains in which the flexibility and innovativeness of the new notion can be productive. In a double sense. By providing and furnishing tools for treating in a quantitative way new domains of the world and, conversely, by receiving from these same uses indications for developing the theory along new directions.

New possible developments (and uses) of fuzzy sets can then be seen which preserve all the freshness of the basic, concept. Some will be outlined in the related contribution [2], which summarize what has been recently proposed in a few papers. What is interesting to 
stress is that under this new challenging way of looking at fuzzy sets new important and fruitfully applications can be planned and envisaged in domains having to do massively with natural language, cognitive aspects and perhaps also with new questions arising from the massive development of networks. All the loose and "imaginative" connections of this Section have been presented starting from very general remarks. They could be based, however, also on scholarly analyses and reconstructions.

\section{A common framework for cybernetics and fuzzy sets (epistemological and historical links)}

\subsection{Cybernetics}

In his book Extrapolation, Interpolation and Smoothing of Stationary Time Series Wiener pursued the goal of unifying Communication Engineering with the field of Statistical Time Series in theory and practice, and the relevant methods for this purpose included filtering in addition to extrapolation and prediction. Moreover, he fused these methods "into a common technique which, in the opinion of the author, is more effective that other existing technique alone." ([10], p. 9.)

Predicting a time series (or a message) could certainly not simply consist of its constant continuation. This would instead be a matter of statistical prediction, estimating the continuation of the time series (or communication), its most probable future pattern while minimizing random error as Wiener described the problem in greater detail:

"We have a message which is a time series, and a noise which is also a time series. If we seek that which we know concerning the message, which is not bound to a specific origin in time, we shall see that such information will generally be of a statistical nature; and this will likewise be true of our information of the same sort concerning the noise alone, or the noise and the message jointly. While this statistical information will in fact never be complete, as our information does not run indefinitely far back into the past, it is a legitimate simplification of the facts to assume that the available information runs back much further into the past than we are called upon to predict the future. The usual electrical wave filter attempts to reproduce a message "in its purity", when the input is the sum of a message and a noise." ([10], p. 10)

Wiener had been working with the filter problem on a very mathematical abstract level, yet he knew very well that there was quite a lot to do below this level in order to implement his standardization program. He thus referred to the work of appropriate experts, naming his MIT colleague Ernst Adolph Guillemin by name: "The problem of realization takes one into the theory of equivalent networks as developed by Guillemin and others." ([10], p. 22)

The research conducted by Wiener and Guillemin in the late 1940s would be consolidated by members of a new generation of communication engineers, which included in particular the electrical engineer Lotfi A. Zadeh.

During his studies at MIT, Zadeh encountered Wiener's Cybernetics and Shannon's "Mathematical Theory of Communication" [11]. "An Extension of Wiener's Theory of Prediction" was the title of an internal report [12] written in 1949 by Zadeh who was then assistant professor at Columbia University in New York. Soon after finishing his Ph D dissertation, he authored this study, though the by-line of the journal article, which was published shortly thereafter, included the name of his supervisor John R. Ragazzini in addition to his own [13]. In this work, which expanded Wiener's prediction theory, Zadeh indicated that the foundations for his work could be found both with Wiener and with Andrei N. Kolmogorov. Both of them, Wiener and Kolmogorov, had proceeded from this problem: If knowledge about the past and present of the physical system are given, how can its future behavior be predicted? Zadeh generalized Wiener's theory in two ways:

- The signal component of a given time series was separated into two parts, of which the first is a nonrandom function in time that can be represented as a polynomial, while the other part functions as a stationary and statistical random function represented by a given correlation function. In Wiener's theory, by contrast, a non-random portion of the signal occurred only when it consisted of a known function in time.

- The response behavior of the predicting system or the weighting function used to make the prediction should disappear outside of a finite time interval. In Wiener's theory, on the other hand, this time interval was assumed to be infinitely long.

Zadeh showed that determining the weighting function leads to the solution to a modified Wiener-Hopf equation, for which he could provide an explicit solution. This publication was a milestone in the theory of network synthesis. Then Zadeh applied these mathematical methods to problems in communication engineering in order to represent general principles in the transformation of signals and in the characterization of inputoutput relationships in nonlinear systems. The obvious discrepancy between mathematical theory and real communication systems eventually led him to completely new ideas on how to expand the theory in order to apply it. By forming new concepts and using the subsequent results, Zadeh tied directly into Wiener's statistical basis of filter theory but then immediately surpassed it.

If filters that were realized as electrical circuits did not operate according to the mathematical theory, then one must be content with less - optimum filters: "A distinction is made between ideal and optimum filters, the former being defined as filters which achieve a perfect separation of signal and noise. ([14] p. 35) If ideal filtration is not possible, though, which is often the case when the signal is mixed with noise, then one must accept that the filtration can only be incomplete. In such cases, a filter that delivers the best possible approxima- 
tion of the desired signal - and "a particular meaning" of "best approximation" is used here - is called an optimum filter. However, there were usually not enough statistical data to determine an optimum filter by means of statistical calculations. For this reason, Zadeh turned away from statistical methods.

\subsection{Information science}

When different specialties began to emerge within electrical engineering in the 1950 s, various "professional groups" were formed within the tradition-rich Institute of Radio Engineers (IRE), which then also published their own "transactions". The contributions to the "Symposium on Information Theory", which had been held in September of 1950, were published in February 1953 in the first edition of the IRE Transactions on Information Theory. Shannon and Wiener have been members of the founding editorial board of this new journal, and in March and June of 1956, each wrote an editorial to demonstrate their own view on this new technological discipline. Shannon called for readers to bear in mind that, despite all of the popularity Information theory had enjoyed over the previous few years, it was not a "universal remedy" and that they should thus return to serious research and development at the highest scientific levels. The fact that Information Theory had been applied successfully in so many fields was good news, but it also obscured the abstract meaning of these terms: "Indeed, the hard core of information theory is essentially a branch of mathematics, a strictly deductive system." Shannon was pushing back against the interdisciplinary expansion of his mathematical theory, and thus naturally against Wiener's Cybernetics, as well. He concluded by calling upon the future authors of the journal to publish only the clearest and the best efforts: "Research rather than exposition is the keynote, and our critical thresholds should be raised." [15] Three months later, Wiener took the opportunity to respond. His plea was also: "Back to the roots!" His editorial was titled with the question "What is Information Theory?" and he was "pleading in this editorial that Information Theory go back of its slogans and return to the point of view from which it originated: that of the general statistical concept of communication."

With references to the statistical character of the mechanics of Josiah Willard Gibbs and the quantum theory that dominated physics as a whole, Wiener also placed Information Theory in this overall context: "What I am here entreating is that communication theory be studied as one item in an entire context of related theories of a statistical nature, and that it should not lose its integrity by becoming a special vested interest attached to a certain set of slogans and clichés." Wiener was clear in his dismissal of Shannon's "purism": "I hope that these Transactions may encourage this integrated view of communication theory by extending its hospitality to papers which, why they bear on communication theory, cross its boundaries, and have a scope covering the related statistical theories. In my opinion we are in a dangerous age of overspecialization." [16] Shannon's en- treaty to concentrate on the core of Information Theory painted him into a scientific corner; it was much more desirable for the statistically based Information Theory to draw from as many scientific areas as possible. Two years later, when also Zadeh was in the editorial board of that journal, he penned his editorial for the March 1958 edition, he pleaded - as Shannon had done two years earlier - for a critical examination of the situation in his own specialist field. However, in this editorial entitled "What Is Optimal?" for the March 1958 edition of the IRE Transactions on Information Theory he asked the readers how reasonable it actually was to insist on optimal solutions. After all, this approach had only established itself since Wiener had publicized his findings on optimal filters and prediction, and proponents were well on their way "to make a fetish of optimality. If a system is not 'best' in one sense or another, we do not feel satisfied. Indeed, we are apt to place too much confidence in a system that is, in effect, optimal by definition." [17] Finding an optimal system would mean choosing a performance criterion, then specifying a class of acceptable systems according to various conditions with respect to design, costs, etc. and finally accepting one of these systems from the specified class as the "best" with regard to these criteria. Zadeh now doubted that this method was any more sensible than the "relatively unsophisticated approach of the pre-Wiener era". [17] The selection of a single performance criterion leaves all of the other criteria that would likewise contribute to performance evaluation unconsidered.

Zadeh similarly criticized the rational selection of decision functions under uncertainty: "What should be done when the probabilities of the "state of nature" characterizing a problem are not known?" Here he rejected the usual solution methods based on stochastics or game theory: "At present no completely satisfactory rule for selecting decision functions is available, and it is not very likely that one will be found in the foreseeable future. Perhaps all that we can reasonably expect is a rule which, in a somewhat equivocal manner, would delimit a set of 'good' designs for a system. In any case, neither Wiener's theory nor the more sophisticated approaches of decision theory have resolved the basic problem of how to find a 'best' or even a 'good' system under uncertainty [17]. - These were his beginning doubts about traditional mathematics, which had heretofore rarely been challenged as a tool for understanding real systems.

In subsequent years he gradually became aware of the fact that although the descriptions of real systems using the instruments of ordinary mathematics were becoming more and more complicated, they were not appropriate to the real systems. His attempts to characterize adaptive, linear and optimum systems demonstrate his efforts to find good definitions within the framework of conventional mathematical theory. His attempts to describe real systems in a mathematically precise manner failed. There were questions about the problems of pattern recognition which Zadeh was beginning to ponder in terms of the gradual membership of elements to sets. 


\subsection{Fuzzy Sets}

In 1964 Zadeh was supposed to give a talk on pattern recognition in the Wright-Patterson Air Force Base, Dayton, Ohio. During this travel he started thinking about pattern recognition problems and grades of membership of an object to be an element of a class: "For example, suppose that we are concerned with devising a test for differentiating between handwritten letters $O$ and $D$. One approach to this problem would be to give a set of handwritten letters and indicate their grades of membership in the fuzzy sets $O$ and $D$. On performing abstraction on these samples, one obtains the estimates $\tilde{\mu}_{O}$ and $\tilde{\mu}_{D}$ of $\mu_{O}$ and $\mu_{D}$ respectively. Then given a letter $x$ which is not one of the given samples, one can calculate its grades of membership in $O$ and $D$, and, if $O$ and $D$ have no overlap, classify $x$ in $O$ or $D . "$ ([18], p. 30)

Whether Zadeh spoke about this in his lecture in Dayton, Ohio in 1964 can unfortunately no longer be determined $^{3}$. At any rate, within a short period of time he had further developed his little theory of "gradual membership" into an appropriately modified set theory - "Essentially the whole thing, let's walk this way, it didn't take me more than two, three, four weeks, it was not long." 4 He wrote his ideas down and these notes appeared as the (in that time secret classified) memorandum RM-4307PR for the RAND-Corporation in October 1964 under authorship of his close friend Richard Bellman, his collaborator Robert Kalaba, and Zadeh [19]. The paper contains the first definitions of the theory of fuzzy sets in a scientific text.

However, his first article in a scientific journal was "Fuzzy Sets" that appeared in Information and Control in June 1965. In contrast to conventional set theory, an object must not either be an element of a set (membership value 1) or not an element of this set (membership value 0 ), but can also have a membership value between 0 and 1 . Therefore, he defined fuzzy sets by their membership function $\mu$, which is allowed to assume any value in the interval $[0,1]$, instead of by their characteristic function, which assumes the values 0 or 1 only [1]. Regarding fuzzy sets $A, B$ in any universe of discourse $X$, Zadeh defined (for all $x \in X$,):

- $A=B$ if and only if $\mu_{A}(x)=\mu_{B}(x)$,

- $A \subseteq B$ if and only if $\mu_{A}(x) \leq \mu_{B}(x)$,

- $\neg A$ is $A$ 's complement $\Leftrightarrow \mu \neg A(x)=1-\mu_{A}(x)$,

- $A \cup B \Leftrightarrow \mu_{A \cup B}(x)=\max \left(\mu_{A}(x), \mu_{B}(x)\right)$,

- $A \cap B \Leftrightarrow \mu_{A \cap B}(x)=\min \left(\mu_{A}(x), \mu_{B}(x)\right)$.

The definition of the first fuzzy operators for the union and intersection of fuzzy sets were influenced from Zadeh's earlier works on electrical filters. Zadeh regarded his theory of fuzzy systems as a general system theory that could be used to cope with the so-called input-output analysis of systems.

\footnotetext{
${ }^{3}$ No sources exist and Zadeh no longer remembers this lecture exactly and so he did not want to either confirm or rule out this detail.

${ }^{4}$ R. S. Interview with L.A. Zadeh, June 19, 2001, University of California, Berkeley, Soda Hall.
}

His interpretation goes back to Shannon's discovery in the use of electrical circuits to model logical statements. In the case of conventional sets, every set $C$ from family of sets $A_{1}, \ldots, A_{i}, \ldots, A_{n}$ can be combined with one another using the conjunctions $\cup$ und $\cap$ and such that it represents a network of circuits $\alpha_{1}, \ldots, \alpha_{n}(i, j=$ $1, \ldots, n)$. By this logic, $A_{i} \cap A_{j}$ and $A_{i} \cup A_{j}$ are, respectively, series and parallel combinations of the circuits $\alpha_{i}$ and $\alpha_{j}$. For the analogous interpretation in the case of fuzzy sets, Zadeh employed the concept of the sieve. He provided the membership function $\mu_{i}(x)$ of $A_{i}$ at $x$ with a "sieve" $S_{i}(x)$ with mesh size $\mu_{i}(x)$. This interpretation results in immediate and clearly evident correlations of the parallel combinations of sieves $S_{i}(x)$ and $S_{j}(x)$ with $\mu_{i}(x) \vee \mu_{j}(x)$ and of their series combinations with $\mu_{i}(x) \wedge \mu_{j}(x)$. If the reader takes into account that the term "sieve" connotes the meaning of a filter then he may catch the analogy of fuzzy sets and electrical filters as outlined in the first section.

\section{A short detour}

Before concluding, let us do a brief detour which can help us in clarifying better the point we are trying to focus in this and the related [2] paper. In the last Section it was evident that the path leading Lotfi Zadeh to conceiving the idea of fuzzy set is strictly related to his "original" way of connecting stimuli, indications and suggestions coming from different sources. That always happens with every investigation and every scientist. The novelty comes when the stimuli belong to very different sources (and the "sources" do not belong to the normal and recognized tradition of an established discipline). Perhaps this is one of the mechanisms through which innovation and creativity come out. This is what emerges in Zadeh's case. His cultural background was different (and broader) than the one of a typical electrical engineer. And this is what makes a comparison possible on the cultural backgrounds of the emerging of both cybernetics and fuzziness as a scientific notion. The originality of Cybernetics is not coinceivable without the multifaceted personality of Wiener and his cultural background. We refer here to the profound analysis done by Leone Montagnini in [20], a book, unfortunately not still translated into english. We refer to [21, 22], reviews in english of the book, for a quick idea of its content. Wiener starting from his very complex cultural vision was able to outline the main features of "information society", this however was not done by a sort of a purely sociological operation (although it was also this see [23]), but outlining the main features of a new science which should play, for the notion of information, the same unifying role that galilean/newtonian mechanics had played for the notion of "motion". As we tried to outline in the previous pages, this was not an easy task. And, in a sense, its big and great innovativeness was payed by a difficulty in being accepted as a "traditional" discipline by the scientific community. However the innovative ideas and provocative questions posed to the researchers in fields having to do with the notion of 
information, has allowed to construct a paradigm of research which has helped the elaboration of new ideas. A deepening of these problems can help in understanding better the potentialities of these new ways of affording questions in a scientific mood but extending some of the rigidities of the path followed in traditional disciplines having a longer history.

\section{Preliminary Conclusions}

In the previous pages we have shown that revisiting some old problems can provide new, interesting, ways of affording present day crucial questions. As we wrote in the Introduction, any conclusion (especially in this kind of papers) cannot but be provisional, but we are strongly convinced that these kind of analyses can help us in doing real progresses in the specific technical investigations at least for one single reason. They allow to work in unknown territories with some general indications which can help us in not doing again (in their entirety) the mistakes done in past decades and, consequently, to begin following really new paths. This discussion will be furthered up, with an outlook of what the future will (and should) bring for fuzziness and cognition in lieu of the contribution of cybernetics in [2].

\section{Acknowledgements}

The work of one of the authors (ET) has been partially funded by the Foundation for the Advancement of Soft Computing (Mieres, Spain), and by the Spanish Government's project MICIIN/TIN 2011-29827-C02-01.

The work of other two authors (ST and MET) has been supported by P.O. F.E.S.R Sicilia 2007-2013 Progetto POSING - Investiamo nel vostro futuro - l'Europa cresce in Sicilia.

\section{References}

[1] Lotfi A Zadeh. Fuzzy sets. Information and control, 8(3):338-353, 1965.

[2] Enric Trillas, Settimo Termini, Marco Elio Tabacchi, and Rudolf Seising. Fuzziness, cognition and cybernetics: an outlook on future. In in this volume, 2015.

[3] Rudolf Seising. Driving technology's future: On the ieee 2014 conference on norbert wiener in the 21 st century, 2014.

[4] Rudolf Seising. On the absence of strict boundaries-vagueness, haziness, and fuzziness in philosophy, science, and medicine. Applied soft computing, 8(3):1232-1242, 2008.

[5] Rudolf Seising. Cybernetics, system (s) theory, information theory and fuzzy sets and systems in the 1950s and 1960s. Information Sciences, 180(23):4459-4476, 2010.

[6] Settimo Termini. A thread through challenging problems. in preparation, 2015.

[7] Guglielmo Tamburrini and Settimo Termini. Do cybernetics, system science and fuzzy sets share some epistemological problems. i. an analysis of cybernetics. In A General Survey of Systems Methodology. Proceedings of the 26th Annual Meeting of the Society for General System Research, Washington, D. C., 1982.

[8] Settimo Termini. Remarks on the development of cybernetics. Scientiae Mathematicae Japonicae, 64(2):461-468, 2006.

[9] Settimo Termini. Imagination and rigor: their interaction along the way to measuring fuzziness and doing other strange things. In S. Termini, editor, Imagination and Rigor, pages 157-176. Springer, 2006.

[10] Norbert Wiener. Extrapolation, interpolation, and smoothing of stationary time series, volume 2. MIT press Cambridge, MA, 1949.

[11] Claude Elwood Shannon. A mathematical theory of communication. ACM SIGMOBILE Mobile Computing and Communications Review, 5(1):355, 2001.

[12] Lotfi A. Zadeh:. An extension of wiener's theory of prediction, research and development report. SPD, 243 The M W Kellogg Company, Special Projects Department, Jersey City, New Jersey, 1949.

[13] Lotfi A Zadeh and John R Ragazzini. An extension of wiener's theory of prediction. Journal of Applied Physics, 21(7):645-655, 1950.

[14] Lotfi A Zadeh. Theory of filtering. Journal of the Society for Industrial and Applied Mathematics, 1(1):35-51, 1953.

[15] Claude E. Shannon. The bandwagon, 1956.

[16] Norbert Wiener. What is information theory?, 1956.

[17] Lotfi A Zadeh. What is optimal. IRE Transactions on Information Theory, 4(1):3, 1958.

[18] Lotfi A. Zadeh. Fuzzy sets and systems. In J. Fox, editor, System Theory. Micro-wave Res. Inst. Symp. Ser. XV, Brooklyn, New York: Polytech., 1965.

[19] Richard Bellman, Robert Kalaba, and L Zadeh. Abstraction and pattern classification. Journal of Mathematical Analysis and Applications, 13(1):17, 1966.

[20] Leone Montagnini. Le armonie del disordine: Norbert Wiener matematico-filosofo del Novecento, volume 43. Istituto Veneto di Scienze, Lettere ed Arti, Venezia, 2005.

[21] Luigi Pepe. Review. MathSciNet - American Mathematical Society, 2010.

[22] Gregor Nickel. Review. Zentralblatt Math of European Mathematical Society, 2010.

[23] Leone Montagnini. Norbert wiener. il matematico che avvistò il nostro tempo. Scienza in Rete, available online at http://www.scienzainrete.it, 2014. 